\title{
Impactos do Offloading de Processamento no Tempo de Execução e Consumo Energético de Dispositivos Móveis
}

\author{
Gabriel B. dos Santos ${ }^{1}$, Fernando A. M. Trinta ${ }^{1}$ e Paulo A. L. Rego ${ }^{1}$ \\ ${ }^{1}$ Group of Computer Networks, Software Engineering and Systems (GREat) \\ Universidade Federal do Ceará (UFC) - Fortaleza, CE - Brasil \\ \{gabrielsantos, fernandotrinta\}@great.ufc.br, pauloalr@ufc.br
}

\begin{abstract}
With the goal of simplifying the design and development of applications that use computation offloading, we developed the framework CAOS D2D to provide an abstraction layer for dealing with low-level tasks related to offloading of methods between Android mobile devices. This paper presents experiments performed to evaluate different aspects of the CAOS D2D framework, such as execution time and power consumption of devices during method offloading. Besides, we also performed experiments to evaluate the applications dependency deployment. The experiments show that computation offloading improves execution times by up to $78 \%$ and reduces power consumption by up to $88 \%$ in comparison to local executions of the same methods.
\end{abstract}

Resumo. Com o objetivo de facilitar o projeto e desenvolvimento de aplicações que usufruam do offloading de processamento, o framework CAOS D2D foi desenvolvido para abstrair tarefas de baixo nível relacionadas ao offloading de métodos entre dispositivos móveis Android. Este trabalho apresenta experimentos realizados para avaliar diferentes aspectos do framework CAOS D2D, como o tempo de execução de tarefas e o consumo de energia de dispositivos durante o offloading de métodos. Além disso, também foram realizados experimentos para avaliar o procedimento de deploy de dependências de aplicações. Os experimentos mostraram que o offloading de processamento proporcionou melhorias de tempos de execução de até $78 \%$ e economia de energia de até $88 \%$, com relação às execuções locais dos mesmos métodos.

\section{Introdução}

O uso de tecnologias digitais está cada vez mais presente na sociedade mundial, e o uso da computação móvel tem aumentado consideravelmente nos últimos anos. Em 2014, o número de usuários de dispositivos móveis ultrapassou o número de usuários de microcomputadores, indicando também que usuários tendem a passar mais tempo conectados à Internet através de dispositivos móveis do que de desktops [Chaffey 2016]. Essa tendência é fortemente representada pelo aumento considerável no uso de aplicativos móveis com acesso à Internet por praticamente todas as camadas sociais. Aplicativos como WhatsApp Messenger tornaram-se quase imprescindíveis para comunicação em nosso cotidiano. Essa massificação foi conquistada a partir da popularização dos smartphones, um modelo simplificado de aquisição de aplicações facilitado a partir das lojas virtuais, e da diversidade de aplicações como jogos em tempo real, redes sociais, dentre outras.

Porém, ao mesmo tempo que se tornam populares, as aplicações tornam-se mais complexas, quer seja por utilizar um número cada vez maior de sensores do dispositivo, 
ou por manipular mais dados heterogêneos e complexos. Além disso, dispositivos móveis precisam ser leves para serem carregados pelos usuários, e com isso, possuem limitações com relação aos seus recursos de processamento e armazenamento.

Por mais que haja atualmente uma tendência de crescimento no poder computacional de dispositivos móveis, características como peso, tamanho, e limitações de bateria tornam intrínseco aos dispositivos móveis o fato de não serem tão computacionalmente capazes quanto dispositivos estáticos contemporâneos a eles [Satyanarayanan 1993], o que torna pouco recomendável a esses dispositivos realizar certos tipos de tarefas, como processamento de imagens ou reconhecimento de padrões [Abolfazli et al. 2016], principalmente se for levado em consideração a necessidade de poupar o consumo de energia, de modo a aumentar a autonomia de funcionamento do aparelho. Todas estas restrições são ainda mais problemáticas quando usuários se engajam em utilizar aplicações com tendência de uso intensivo de dados e sensores embutidos nos dispositivos [Júnior et al. 2016, Gonçalves et al. 2016]. Exemplos dessas aplicações incluem jogos e realidade aumentada (e.g., Pokémon $\mathrm{Go}^{1}$ ), processamento de imagens e linguagem natural (e.g., Google Translator ${ }^{2}$ ) e computação vestível (e.g., Nike+ Run Club $^{3}$ ).

Uma das possíveis soluções para contornar esse problema é a Computação Móvel em Nuvem (do inglês, Mobile Cloud Computing - MCC). Segundo [Dinh et al. 2013], MCC tem por objetivo provisionar um conjunto de serviços equivalentes ao da nuvem, adaptados à capacidade de dispositivos com recursos restritos, de modo a trazer melhorias de desempenho das aplicações ou mesmo economia de energia nos dispositivos. Em geral isso é alcançado por meio de uma técnica conhecida como offloading, em que processos e/ou dados são transferidos de um dispositivo mais fraco (e.g., smartphone) para um dispositivo mais potente (e.g., máquina virtual na nuvem).

Segundo [Satyanarayanan et al. 2009], a técnica de offloading obtém resultados ainda melhores quando se dá entre dispositivos próximos, já que a latência de comunicação é um dos principais empecilhos para que a migração de tarefas seja mais eficiente [Cuervo et al. 2010, Fernando et al. 2013]. Com isso, propôs-se o conceito de cloudlet, uma infraestrutura para processar tarefas ou armazenar dados em nome de dispositivos mais fracos, mas que encontra-se na mesma rede local do dispositivo móvel. Um cloudlet pode ser um servidor dedicado, um notebook pessoal de um usuário, ou mesmo outro dispositivo móvel que deseje ofertar seus recursos para ajudar dispositivos mais fracos.

O suporte a offloading para dispositivos móveis já foi proposto por vários estudos [Artail et al. 2015, Liao et al. 2015, Teo 2012, Ferrari et al. 2016]. Um deles é o CAOS D2D [Santos et al. 2017], uma plataforma para offloading de métodos entre dispositivos Android. O CAOS D2D permite que o desenvolvedor marque quais métodos são candidatos a migrar para um outro dispositivo, e de acordo com a disponibilidade de servidores aptos para o offloading na rede, o sistema decide quando migrar a tarefa. Nesse contexto, este trabalho tem como objetivo realizar experimentos, utilizando o framework CAOS D2D, para avaliar o impacto do offloading de processamento no tempo de execução de métodos e consumo energético de dispositivos móveis, bem como o procedimento de deploy de dependências de aplicações - uma das característica do framework.

\footnotetext{
${ }^{1}$ http://www.pokemongo.com

${ }^{2} \mathrm{http}: / /$ translate.google.com

${ }^{3}$ http://www.nike.com.br/running/nrc-app
} 
O restante deste artigo está dividido da seguinte forma: a Seção 2 apresenta os trabalhos relacionados, comparando as principais características deles a este trabalho. A Seção 3 apresenta uma visão geral do CAOS D2D, framework utilizado nos experimentos realizados. A Seção 4 discute os experimentos que foram realizados para, além de avaliar impactos do offloading nos tempos de execução e consumo de energia de dispositivos móveis, avaliar também diferentes aspectos do CAOS D2D. E finalmente, a Seção 5 expõe as considerações finais sobre os experimentos e lista os trabalhos futuros.

\section{Trabalhos Relacionados}

Nos últimos anos, uma variedade de estudos propôs o suporte a offloading de processamento de um dispositivo móvel para outros dispositivos móveis [Artail et al. 2015, Liao et al. 2015, Teo 2012, Ferrari et al. 2016]. Esses estudos apresentam diferentes requisitos e abordagens para alcançar os objetivos a que se propõem. A seguir, são apresentados tais trabalhos e suas principais características.

O framework definido em [Artail et al. 2015] oferece suporte a offloading deviceto-device, em um modelo de nuvem federada. Nesse framework, dispositivos móveis dispostos a agir como servidores oferecem serviços para dispositivos próximos dentro da mesma rede, nos moldes de uma nuvem SaaS tradicional, independente de sistema operacional. Para que um aplicativo possa usufruir dos recursos do framework, ele deve ser projetado levando em consideração a modularização entre serviço e cliente de nuvem, o que adiciona certa complexidade ao projeto. Por um lado, a solução conta com mecanismos de privacidade e incentivos; e por outro lado, o framework pressupõe que haja um diretório central de cloudlets móveis dentro da rede, o que pode reduzir a disponibilidade do serviço caso, por exemplo, esse dispositivo esteja sobrecarregado ou fora da rede. Além disso, o consumo de energia do dispositivo que assume o papel de nó raiz aumenta com o passar do tempo.

A abordagem proposta em [Liao et al. 2015] propõe um framework para offloading device-to-device voltado para melhoria do tempo de execução de aplicações, em que o particionamento delas é feito em duas grandes camadas - front-end e back-end. Nesse trabalho, a porção front-end da aplicação sempre é executada no lado cliente, e a porção back-end da aplicação apresenta a possibilidade de ser executada em um dispositivo remoto. Esse framework usa o protocolo OpenFlow implementado em cima de uma rede definida por software para abstrair a comunicação de rede entre os dispositivos. Entretanto, as implementações existentes do OpenFlow para dispositivos móveis são relativamente recentes e enfrentam alguns problemas em dispositivos móveis [Kolias et al. 2013]. Além disso, a divisão da aplicação em duas grandes camadas é um pressuposto para utilização da solução, o que pode exigir alterações de projeto em alguns aplicativos.

O framework Hyrax [Teo 2012] é uma solução de offloading device-to-device voltada para crowdsourcing, com foco na melhoria do tempo de execução de aplicações utilizando uma implementação de Map Reduce derivada do Hadoop. O Hyrax busca dar suporte à formação de clusters compostos por dispositivos Android. Apesar da proposta de crowdsourcing com dispositivos móveis alcançar resultados satisfatórios, Hyrax conta com uma série de pressupostos que nem sempre podem ser cumpridos, como:

- A necessidade de um computador que age como nó central, que não pode falhar. 
- Todos os dispositivos envolvidos no processamento já devem possuir os dados a serem processados de antemão.

- Os dados não podem sofrer alterações durante o processamento.

Além dos pressupostos de projeto, para utilizar o Hyrax, deve-se implementar as funções Map e Reduce - cerne do paradigma MapReduce - para as funcionalidades que devem ter as melhorias de tempo de execução.

A arquitetura Anyrun Computing (ARC) [Ferrari et al. 2016] propõe um modelo de offloading métodos dinâmico e oportunista entre dispositivos Android, com foco em melhoria do tempo de execução e economia de energia. Para utilizar o ARC, o desenvolvedor deve refatorar os códigos-fonte, de maneira que as classes do ARC sejam usadas para realizar as chamadas dos métodos candidatos a offloading.

[Ghasemi-Falavarjani et al. 2015] desenvolveram um middleware que coleta informações contextuais para tomada de decisão de offloading com foco na melhoria do tempo de execução e consumo de energia. Um protótipo foi implementado para a plataforma Android, onde é possível fazer offloading para dispositivos móveis. O protótipo foi desenvolvido para uma aplicação de processamento de imagem, onde foi preciso desenvolver código do cliente e do servidor, não sendo, assim, fácil a adaptação em outras aplicações/cenários

O HyMobi [Flores et al. 2017] é um framework para offloading D2D em que os dispositivos móveis estão organizados em uma espécie de "rede social", em que cada dispositivo (dispositivo móvel, cloud ou cloudlet) possui um perfil e pontuação, sobre os quais as decisões de offloading são tomadas. O particionamento de aplicações é a nível de métodos, que devem ser adaptados para que sejam utilizados os recursos do framework. Essa adaptação expõe ao desenvolvedor aspectos da reflexão utilizada pela API, o que pode ser um ponto para surgimento de bugs.

A Tabela 1 apresenta um resumo das principais características de cada trabalho, com relação a: sistema operacional, foco de melhoria da solução, abordagem de particionamento de aplicações e nível de esforço para adaptação de aplicativos.

O esforço para adaptar aplicativos de forma a usarem as funcionalidades de cada solução foi classificado como:

Baixo: Quando as alterações em código-fonte se resumem a adicionar bibliotecas e marcar os métodos passíveis de offloading com anotações, sem grandes refatorações.

Médio: Quando o código interno de métodos deve ser refatorado para usar classes do framework, mas sem necessariamente alterar seu comportamento externo.

Alto: Quando para um aplicativo usar a solução proposta, ele deve se encaixar em um paradigma de aplicação específico (front-end e back-end, cliente/servidor ou $M a$ pReduce), o que pode exigir um extenso trabalho de refatoração de código-fonte.

\section{CAOS D2D}

O CAOS D2D (Device-to-Device) [Santos et al. 2017] é uma infraestrutura de software projetada no Grupo de Redes de Computadores, Engenharia de Software e Sistemas da Universidade Federal do Ceará, para auxiliar no desenvolvimento de aplicações móveis e sensíveis ao contexto na plataforma Android. Ele surge como uma versão estendida do CAOS (Context Acquisition and Offloading System) [Gomes et al. 2017], e conta com 
Tabela 1. Comparação das principais características dos trabalhos citados

\begin{tabular}{|c|c|c|c|c|}
\hline Trabalho & $\begin{array}{l}\text { Sistema } \\
\text { operacional }\end{array}$ & Foco de melhoria & $\begin{array}{l}\text { Abordagem de } \\
\text { particionamento }\end{array}$ & $\begin{array}{l}\text { Esforço } \\
\text { para } \\
\text { adaptação }\end{array}$ \\
\hline Artail et. Al (2015) & Multiplataforma & $\begin{array}{l}\text { Tempo de execução e } \\
\text { armazenamento }\end{array}$ & Serviços & Alto \\
\hline Liao, Qiu e Leung (2015) & Multiplataforma & Tempo de execução & $\begin{array}{l}\text { Camadas Front end e } \\
\text { back end }\end{array}$ & Alto \\
\hline Hyrax & Android & Tempo de execução & Map reduce & Alto \\
\hline $\mathrm{ARC}$ & Android & $\begin{array}{l}\text { Tempo de execução e } \\
\text { economia energética }\end{array}$ & Métodos (dinâmico) & Médio \\
\hline Ghasemi-Falavarjani et al. 2015 & Android & $\begin{array}{l}\text { Tempo de execução e } \\
\text { economia energética }\end{array}$ & Não se aplica* & Alto \\
\hline HyMobi & Android & $\begin{array}{l}\text { Tempo de execução e } \\
\text { economia energética }\end{array}$ & Métodos (dinâmico) & Médio \\
\hline CAOS D2D & Android & $\begin{array}{l}\text { Tempo de execução e } \\
\text { economia energética }\end{array}$ & $\begin{array}{l}\text { Métodos } \\
\text { (estático e dinâmico) }\end{array}$ & Baixo \\
\hline
\end{tabular}

suporte ao mecanismo de offloading para delegar a execução de tarefas de dispositivos móveis Android para outros dispositivos Android.

O principal objetivo do CAOS D2D é fornecer uma camada de abstração de tarefas de baixo nível relacionadas ao offloading, para facilitar o projeto de aplicações que usem deste. Ele suporta o offloading a nível de métodos, que são marcados com uma anotação Java. Além disso, ele possui um mecanismo de descoberta de serviço para identificar dispositivos dispostos a atuar como servidores de processamento disponíveis na mesma rede em que o dispositivo cliente está conectado.

Os componentes do CAOS D2D são executados no dispositivo móvel cliente (CAOS D2D API) e no dispositivo servidor (CAOS D2D Server), como ilustrado na Figura 1. Em sua maioria, os componentes do CAOS D2D são formados por módulos correspondentes - presentes tanto no lado cliente quanto no lado servidor, de forma que esses módulos se comunicam entre si, através da abordagem cliente/servidor.
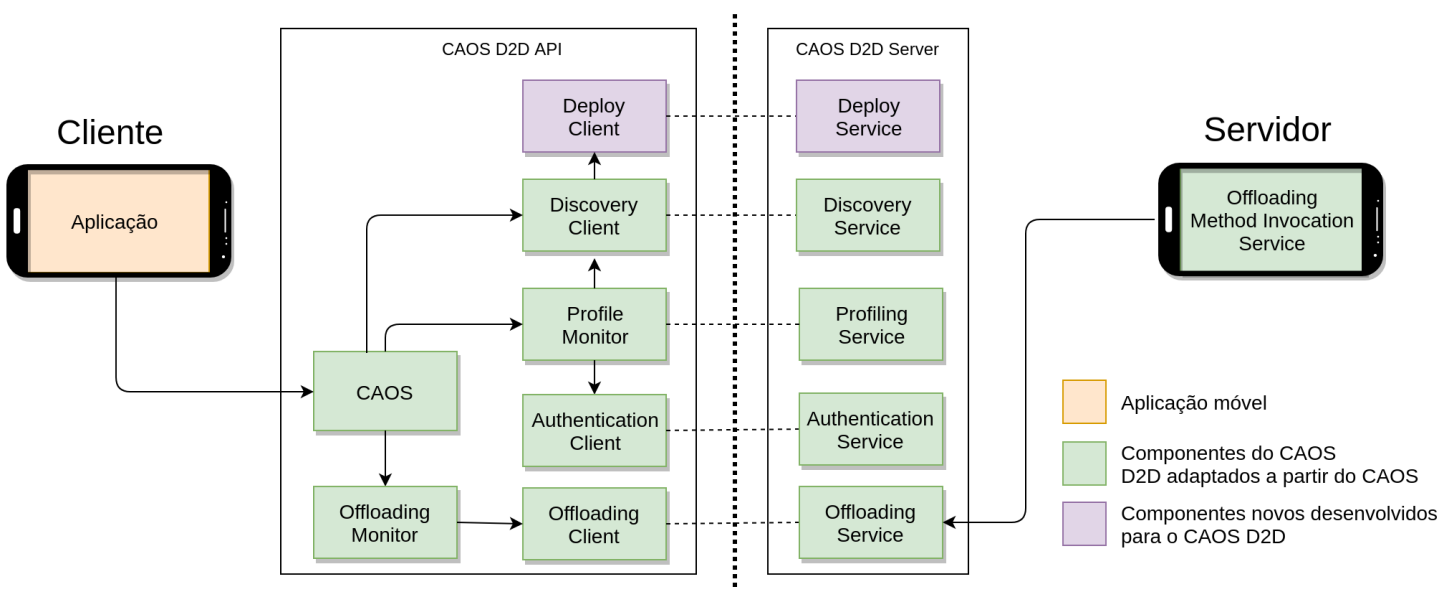

Figura 1. Visão geral da arquitetura do CAOS D2D

Os módulos presentes no CAOS D2D API têm a iniciativa de iniciar todos os fluxos de comunicação da solução. Eles têm a responsabilidade de: 
- Anunciar que o dispositivo está presente na rede, através do Discovery Client;

- Autenticar o dispositivo cliente no servidor, através do Authentication Client;

- Injetar dependências no CAOS D2D Server, por meio do Deploy Client. A dependência é uma cópia do aplicativo móvel (arquivo .APK do Android);

- Fazer monitoramento da qualidade da rede, por meio do Profile Monitor, onde são monitoradas as métricas: taxa de download, upload, latência, dentre outras;

- Iniciar o processo de offloading de um método, por meio do Offloading Client.

O CAOS D2D Server responde às requisições do cliente e tem responsabilidade de:

- Receber requisições do cliente, tratá-las e respondê-las apropriadamente;

- Manter-se visível para dispositivos clientes, através do Discovery Service; requisições de offloading;

- Por meio do Authentication Service, informar ao dispositivo cliente qual a sua configuração de hardware, estado de bateria e se o servidor possui as dependências necessárias para a aplicação cliente;

- Através do Deploy Service, receber dependências de aplicações de clientes, e armazená-las no sistema de arquivos do dispositivo servidor, para ficar apto a realizar o offloading para essas aplicações;

- Através do Offloading Service, receber requisições de offloading, processar os métodos sob demanda, e retornar seus resultados.

No dispositivo móvel servidor, após a inicialização do aplicativo CAOS D2D Server, todos os módulos que hospedam serviços permanecem de prontidão, no aguardo de uma mensagem enviada pelos módulos clientes que estiverem em execução no CAOS D2D API, em algum outro dispositivo móvel.

\section{Planejamento e Resultados dos Experimentos}

Com o objetivo de avaliar os impactos do offloading de processamento no consumo de energia e tempo de execução de métodos de aplicações, bem como validar as funcionalidades e módulos do CAOS D2D, foram planejados experimentos para realizar medições desses aspectos em aplicações que foram configuradas para utilizar o framework. Em resumo, os experimentos foram realizados com os seguintes propósitos:

- Obter resultados sobre impactos de tempo de execução e economia de energia durante o offloading de processamento;

- Mensurar o consumo de energia e o tempo decorrido durante a implantação de dependências de aplicações do cliente para o servidor (deploy);

Para medir o tempo de execução, os tempos foram coletados com o auxílio de logs de aplicações fornecidos pela plataforma Android. Já a métrica usada para avaliação do consumo de energia pelos dispositivos móveis foi calculada através de medições feitas no aparelho Monsoon Power Monitor ${ }^{4}$. O aparelho é instalado nos contatos da bateria do dispositivo móvel, agindo entre a bateria e o dispositivo móvel. Através de um software fornecido pelo fabricante, o Power Monitor age como uma "bateria configurável", em que fatores como voltagem e capacidade podem ser definidos pelo usuário, e também

\footnotetext{
${ }^{4}$ https://www.msoon.com
} 
fornece dados em tempo real sobre o consumo de energia, potência, voltagem, corrente, e estimativa de tempo de duração da "bateria".

Como o Power Monitor coleta o consumo de todo o dispositivo, sem discriminar por aplicativo, os processos em segundo plano foram finalizados durante os testes, mantendo em execução apenas os aplicativos diretamente envolvidos nos experimentos.

\subsection{Avaliação de offloading}

Para avaliar os tempos de execução de tarefas e o consumo de energia, experimentos foram realizados em diferentes cenários com duas aplicações Android, configuradas com o CAOS D2D API para permitir o offloading de métodos.

Os dispositivos Android utilizados nessa fase foram: um smartphone LG G3 Beat, aqui referenciado como "Handset A"; um smartphone Motorola Moto G4 Play, chamado aqui de "Handset B", e um aparelho do tipo "Android TV Box", aqui chamado de "TV Box". A Tabela 2 resume a configuração dos equipamentos utilizados. Os dispositivos foram conectados à mesma rede sem fio 802.11 n, através de um ponto de acesso TPLink TL-WA901ND. A rede estava isolada, de forma que apenas os dispositivos móveis utilizados nos testes estavam conectados à rede.

Tabela 2. Descrição dos equipamentos utilizados

\begin{tabular}{ll}
\hline Dispositivo & Configuração \\
\hline Handset A & Android 4.4.2, 1 GB de RAM, Qualcomm Snapdragon 400 (Cortex A7, 1.2 GHz 4 core) \\
\hline Handset B & Android 6.0.1, 2 GB de RAM, Qualcomm Snapdragon 410 (Cortex A53, 1.2 GHz 4 core) \\
\hline TV Box & Android 6.0, 1 GB RAM, Amlogic S905X (Cortex-A53 $2 \mathrm{GHz} 4$ core) \\
\hline
\end{tabular}

Um dos aplicativos escolhidos para os testes é o Matrix Operations, que gera matrizes quadradas com valores aleatórios, e mede o tempo decorrido em operações de soma e multiplicação. Esse aplicativo foi escolhido para os testes por ilustrar 2 cenários distintos quanto ao comportamento do custo computacional de métodos: a soma de matrizes é uma operação de baixo custo computacional; considerando-se $N$ o número de elementos da matriz, a complexidade da adição é calculada como $O(N)$; enquanto a multiplicação de matrizes é uma operação de custo mais alto, com complexidade calculada como $O\left(N^{\frac{1}{5}}\right)$. O uso desses dois métodos, com diferentes tamanhos de argumentos, pode representar casos em que o offloading se mostre uma opção favorável e também pode representar casos em que o custo de transferir grandes argumentos pela rede não compensem a economia de energia ou de tempo proporcionados pelo offloading. Durante os testes, foram executadas operações de soma e multiplicação de matrizes quadradas de tamanho 200x200 e 800x800.

O outro aplicativo utilizado nos testes é o Camera Offloading, um aplicativo de processamento de imagens que permite ao usuário tirar uma foto com a câmera e aplicar filtros de imagens da biblioteca PhotoFilter ${ }^{5}$. Dois filtros foram utilizados nos testes: Snow e Emboss. Esses filtros foram escolhidos por representarem dois exemplos distintos: enquanto o filtro Snow é de baixo custo computacional, com execução rápida, mesmo localmente; o filtro Emboss tem de custo computacional mais elevado, devido ao fato de ele ser uma combinação de outros filtros. Durante os experimentos, os dois filtros foram

\footnotetext{
${ }^{5}$ https://github.com/mukeshsolanki/photofilter
} 


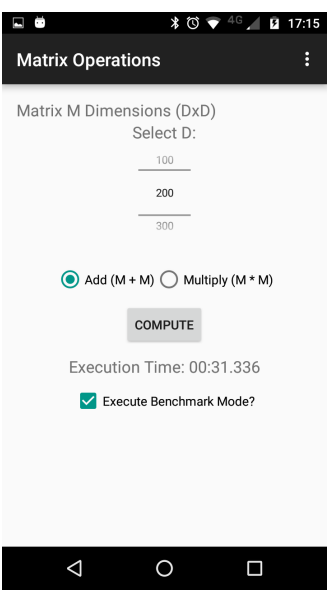

(a)

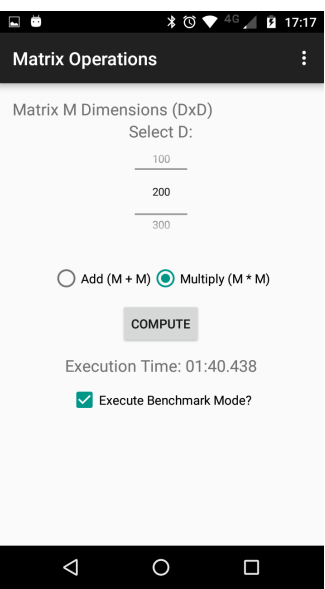

(b)

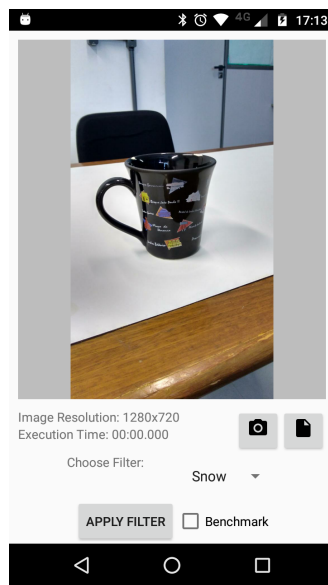

(c)

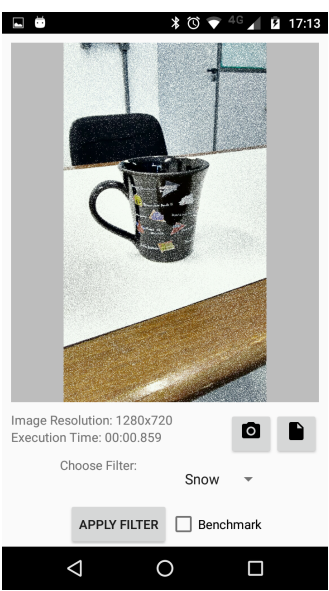

(d)

Figura 2. Capturas de tela dos aplicativos utilizados, tiradas no Handset B. Matrix Operations, (a) após realizar 30 operações de soma, e (b) 30 operações de multiplicação, com matrizes $200 \times 200$. Camera Offloading, (c) antes e (d) após aplicar o efeito Snow em uma foto de $1 \mathrm{MP}$

utilizados com fotos de 1,6MP. Os dois aplicativos utilizados nos testes são ilustrados na Figura 2.

Para esses experimentos, foram considerados três grandes cenários de testes: no cenário $\mathrm{C} 1$, o Handset $\mathrm{B}$ atua como cliente interessado no offloading, e o Handset A atua como servidor. No cenário $\mathrm{C} 2$, o Handset $\mathrm{B}$ age como cliente e o TV Box faz o papel de servidor. E no cenário $\mathrm{C} 3$, o Handset $\mathrm{B}$ executa os métodos localmente (cenário de controle, que serve de base de comparação com os outros dois cenários).

Nos três cenários, foram realizados os mesmos procedimentos: No aplicativo Matrix Operations, foi realizada a soma e a multiplicação de matrizes, com dimensão 200x200 e 800x800, resultando em doze diferentes casos. No aplicativo Camera Offloading, foram aplicados os filtros Snow e Emboss em imagens de 1,6MP, resultando em 6 casos. No total, foram realizados 18 casos, repetidos trinta vezes, o que totaliza 540 execuções de métodos avaliadas. Durante os três experimentos, houve uma pausa programada de 1 segundo entre todas as execuções de métodos, e o consumo de energia do Handset $\mathrm{B}$ foi medido com o Power Monitor. As medições foram feitas para, além de coletar métricas sobre tempos de execução de métodos, medir o consumo de energia do Handset $\mathrm{B}$ quando ele atua como cliente e quando executa os processamentos localmente.

A seguir, são analisados os dados relacionados aos tempos de execução de métodos e consumo de energia por parte do Handset $\mathrm{B}$, nos casos possíveis no que diz respeito a offloading: quando ele realiza offloading para o Handset A, para o TV Box, e quando ele executa as tarefas localmente.

\subsubsection{Avaliação de tempo de execução}

A Figura 3 apresenta as médias de tempo de execução dos métodos para os diferentes casos analisados. Ao analisar os resultados, chega-se às seguintes conclusões. Para realizar o 
offloading de um método, o CAOS D2D encapsula o método e seus argumentos em um objeto a ser transmitido pela rede. Pelo fato da transmissão desse objeto ter o seu custo de tempo, nota-se que operações de baixo custo computacional não se beneficiam com o offloading em termos de tempo de execução.

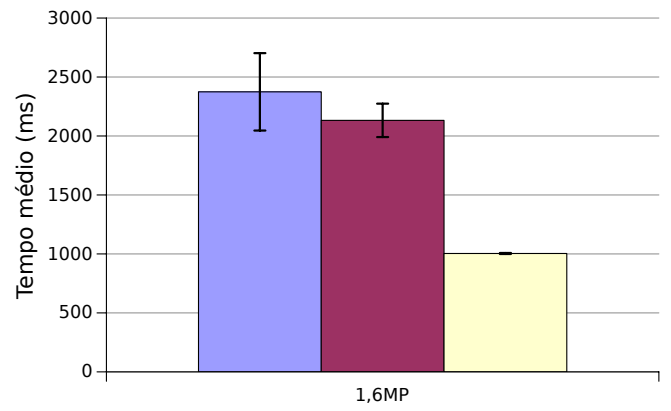

(a) Camera Offloading: Snow

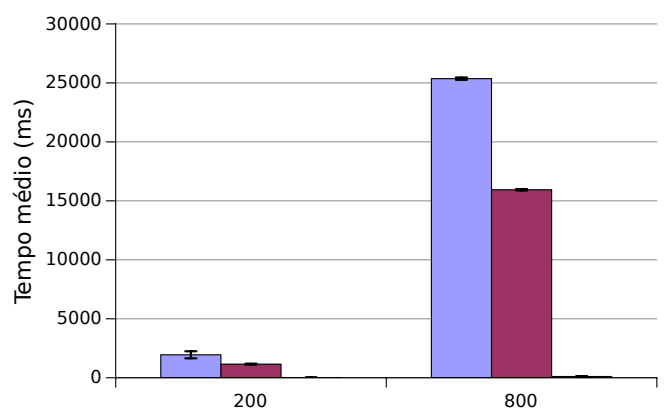

(c) Matrix Operations: Soma

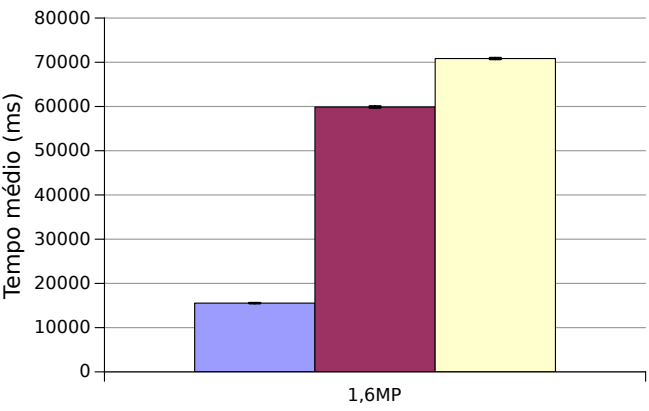

(b) Camera Offloading: Emboss

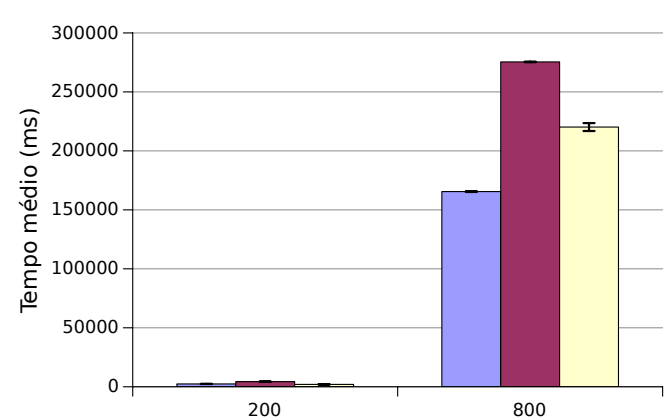

(d) Matrix Operations: Multiplicação

Offloading para o Handset A

Offloading para o TV Box

$\square$ Execução local (sem offloading)

Figura 3. Tempos médios de operações avaliadas nos testes de offloading

No caso do aplicativo Matrix Operations, a execução local da soma de matrizes de dimensão 800x800, por exemplo, foi $99,6 \%$ mais rápida do que quando foi executada no Handset A e 99,3\% mais rápida do que quando foi executada no TV Box. Isso se deve ao fato do objeto transmitido pelo CAOS D2D para realização do offloading ser, nesse caso em especial, 5,12MB; e o objeto representando o resultado dessa soma ter em média 2,6MB. O tempo necessário para transmissão desses dois objetos fez grande diferença frente ao tempo empregado na operação de soma propriamente dita, que é de baixo custo.

No caso do aplicativo Camera Offloading, nota-se que o Handset B teve melhores tempos de execução quando aplicou o efeito menos custoso - Snow - localmente. A execução local desse efeito foi em média 57,7\% mais rápida do que no Handset A, e 52,9\% mais rápida do que no TV Box.

Em termos de tempos de execução, o offloading se mostrou uma opção mais proveitosa para casos em que o custo de execução maior apresenta um peso mais significativo no processo de offloading do que o custo de transmissão dos argumentos através da rede. No aplicativo Camera Offloading, a aplicação do efeito Emboss com offloading para o Handset A foi 78,1\% mais rápida, e para o TV Box foi 15,5\% mais rápida, ambos os casos em comparação com a a execução local. 
No aplicativo Matrix Operations, a multiplicação de matrizes de dimensão 800x800 no TV Box foi em média $25,1 \%$ mais lenta do que quando foi executada localmente; diferente da multiplicação no Handset A, que foi $24,8 \%$ mais rápida do que na execução local. Essa tendência continua também com a multiplicação de matrizes de tamanho 200x200. Essa diferença de comportamento para diferentes métodos também pode ser observada no aplicativo Camera Offloading: a aplicação do efeito Snow em offloading para o TV Box foi mais rápida do que para o Handset A, porém a aplicação do efeito Emboss para o Handset A foi mais rápida do que para o TV Box.

Esses casos indicam que um dispositivo que executa uma determinada tarefa mais rápido do que outros nem sempre será a melhor opção para o offloading.

\subsubsection{Avaliação de consumo de energia}

Os dados acerca do consumo energético nos experimentos estão organizados na Tabela 3. Partindo da análise desses dados, pode-se observar que o consumo de energia por parte do Handset $\mathrm{B}$ foi em geral proporcional ao tempo de execução das tarefas. Adicionalmente, medições foram realizadas no Handset B para aferir seu consumo de energia em modo idle, apenas com a tela ligada. Em 77,42s a potência média mensurada foi de $532,95 \mathrm{~mW}$.

\begin{tabular}{|c|c|c|c|c|c|}
\hline Cenário & Aplicativo & Método & Argumento & $\begin{array}{l}\text { Potência } \\
\text { média } \\
(\mathrm{mW})\end{array}$ & $\begin{array}{c}\text { Energia média } \\
\text { consumida por } \\
\text { método }(\mathrm{J})\end{array}$ \\
\hline \multirow{5}{*}{$\mathrm{C} 1$} & Camera Offloading & emboss & $1.6 \mathrm{MP}$ & 594.34 & 9.24 \\
\hline & \multirow{4}{*}{ Matrix Operations } & \multirow{2}{*}{ add } & $200 \times 200$ & 1006.41 & 1.96 \\
\hline & & & $800 \times 800$ & 1109.15 & 28.13 \\
\hline & & \multirow{2}{*}{ multiply } & $200 \times 200$ & 858.32 & 2.04 \\
\hline & & & $800 \times 800$ & 612.61 & 101.38 \\
\hline \multirow{5}{*}{$\mathrm{C} 2$} & Camera Offloading & emboss & $1.6 \mathrm{MP}$ & 569.50 & 34.10 \\
\hline & \multirow{4}{*}{ Matrix Operations } & \multirow{2}{*}{ add } & $200 \times 200$ & 978.87 & 1.13 \\
\hline & & & $800 \times 800$ & 1364.62 & 21.75 \\
\hline & & \multirow{2}{*}{ multiply } & $200 \times 200$ & 751.49 & 3.26 \\
\hline & & & $800 \times 800$ & 590.16 & 162.54 \\
\hline \multirow{5}{*}{$\mathrm{C} 3$} & Camera Offloading & emboss & $1.6 \mathrm{MP}$ & 1135.17 & 80.42 \\
\hline & \multirow{4}{*}{ Matrix Operations } & \multirow{2}{*}{ add } & $200 \times 200$ & 978.87 & 0.01 \\
\hline & & & $800 \times 800$ & 834.97 & 0.09 \\
\hline & & \multirow{2}{*}{ multiply } & $200 \times 200$ & 910.78 & 1.81 \\
\hline & & & $800 \times 800$ & 1101.38 & 242.52 \\
\hline
\end{tabular}

Tabela 3. Cálculos da energia média consumida em operações avaliadas nos testes de offloading

Nos casos em que o offloading proporcionou melhoria no tempo de execução de tarefas, ele também proporcionou melhoria no consumo de energia. No aplicativo Camera Offloading, a aplicação do efeito Emboss apresentou economia de 88,5\% quando feito em offloading para o Handset A, e economia de 57,6\% quando realizado em offloading para o TV Box. A execução do filtro em offloading representou para o Handset B um aumento na potência elétrica média de $11,5 \%$ em $\mathrm{C} 1$ e de apenas $6,9 \%$ em $\mathrm{C} 2$, com relação ao modo idle. Para efeito de comparação, em C3 (onde a execução do método foi local) 
o aumento de potência elétrica foi de $113 \%$. a caso do aplicadtivo Matrix Operations, a multiplicação de matrizes de dimensão $800 x 800$, por exemplo, se mostrou 58,2\% mais econômica em termos de consumo de energia quando realizada em offloading para o Handset A. Nesse caso, o aumento da potência elétrica média foi de $15 \%$ com relação ao modo idle. E, diferente do comportamento observado quanto ao aspecto de tempos de execução, a mesma tarefa também se mostrou vantajosa quando realizada em offloading para o TV Box. Nesse caso, a economia foi em média de 33\%, com aumento de potência com relação ao modo idle de 10,7\% (para comparação, em C3, essa tarefa demanda um aumento de $106,7 \%$ na potência elétrica média). Essa divergência entre o tempo decorrido e a energia consumida se deve ao fato de que em boa parte do tempo empregado pelo Handset B durante a operação de offloading, o dispositivo móvel se encontra "aguardando" o resultado do processamento, em vez de efetivamente realizando a tarefa, e portanto, poupando energia. Por esse motivo, pode-se considerar que, no aspecto de melhora no consumo, o offloading é mais proveitoso à medida que a tarefa é mais custosa.

Por outro lado, nos casos em que a tarefa migrada tem custo computacional baixo, percebe-se que a execução local das tarefas representa uma opção melhor em termos de consumo de energia. No aplicativo Camera Offloading, a aplicação do efeito Snow em offloading para o Handset A apresentou em média consumo 93,7\% maior do que na execução local, e consumo 77,1\% maior quando realizada em offloading para a TV Box. Para a Matrix Operations, a soma de matrizes de dimensão 800x800, quando executado localmente, apresentou consumo 99,7\% menor do que ao fazer offloading para o Handset A, e 99,6\% menor do que realizando o offloading para a TV Box.

\subsection{Avaliação de deploy de dependências}

Para realizar os experimentos com finalidade de avaliar o consumo energético e o tempo gasto pelo módulo de deploy de dependências do CAOS D2D, foram utilizados os mesmos dispositivos Handset A e Handset B, utilizados para os experimentos de avaliação de offloading. Esses dispositivos foram conectados à uma rede sem fio $802.11 \mathrm{n}$, provido através de um computador que foi configurado para atuar como um ponto de acesso, para que se pudesse ter controle sobre a velocidade máxima da rede ao qual os dois dispositivos móveis estavam conectados.

Para a realização desses experimentos, foram desenvolvidos dois aplicativos configurados com o CAOS D2D, a partir de variações do aplicativo Camera Offloading, mencionado anteriormente. Um deles - chamado aqui de "App A"- tem no seu arquivo APK 1.830.413B, ou 1,83MB (ou 1,74MiB). O outro aplicativo - referenciado aqui como “App B" - tem em seu APK 54.251.284B, ou 54,25MB.

Os experimentos para avaliação do módulo de deploy de dependências seguiram o seguinte roteiro: Para cada um dos dois aplicativos - App A e App B - foram realizadas operações de deploy de dependências do Handset B para o Handset A, 30 vezes com a velocidade máxima da rede configurada para $8 \mathrm{mbps}$, e mais 30 vezes com a velocidade de rede em $4 \mathrm{mbps}$. Foi adicionado também um intervalo de 1 segundo entre as execuções individuais. Durante os experimentos, foram coletados dados sobre o tempo decorrido para as operações de deploy de dependências através de logs extraídos do Handset $\mathrm{B}$, e foram coletados dados sobre o consumo de energia do Handset B, através do Power Monitor.

Para realizar o deploy de dependências no dispositivo servidor, o CAOS D2D envia 
o arquivo APK do aplicativo no qual está sendo usado, em vez de um arquivo dentro do diretório de recursos do aplicativo. Por isso, espera-se que o tempo necessário para deploy de dependências dependa, além da velocidade da rede na qual o dispositivo móvel cliente está, também do tamanho do arquivo APK do aplicativo que está usando o CAOS D2D.

Os resultados obtidos nos experimentos descritos estão organizados na Tabela 4. Partindo da observação desses dados, pode-se perceber que, primeiramente, os tempos decorridos para transmitir os arquivos APK pela rede são próximos dos tempos ideais calculados a partir dos tamanhos dos arquivos APK e da largura de banda da rede. As perdas médias se situaram entre 5,3\% e 10,2\% acima do tempo ideal, sendo que essas diferenças se tornam menos significativas à medida que o tamanho do arquivo APK aumenta.

Observando a análise de variância na Tabela 5, nota-se que os fatores tamanho do arquivo a ser transmitido, a velocidade de rede e tempo decorrido para transmissão têm impacto na energia consumida pelo dispositivo móvel cliente.

Tabela 4. Dados coletados durante os experimentos para avaliação do módulo de deploy de dependências

\begin{tabular}{ccccccrrr}
\hline $\begin{array}{c}\text { Velocidade de } \\
\text { Rede (mbps) }\end{array}$ & $\begin{array}{c}\text { Tamanho } \\
\text { do APK (B) }\end{array}$ & $\begin{array}{c}\text { Potência } \\
\text { Média (mW) }\end{array}$ & $\begin{array}{c}\text { Tempo } \\
\text { Ideal (ms) }\end{array}$ & $\begin{array}{c}\text { Média } \\
\text { tempo (ms) }\end{array}$ & $\begin{array}{c}\text { IC } \\
\text { Tempo } \\
(95 \%)\end{array}$ & $\begin{array}{c}\text { Diferença } \\
\text { Tempo } \\
\text { Ideal (\%) }\end{array}$ & $\begin{array}{c}\text { Média } \\
\text { Energia (J) }\end{array}$ & $\begin{array}{c}\text { IC } \\
\text { Energia } \\
(95 \%)\end{array}$ \\
\hline \multirow{2}{*}{4} & 1830413 & 677.79 & 3660.83 & 3943.77 & 58.17 & 7.17 & 2.67 & 0.04 \\
\cline { 2 - 9 } & 54251284 & 667.44 & 108502.57 & 114538.63 & 373.67 & 5.27 & 76.45 & 0.25 \\
\hline \multirow{2}{*}{8} & 1830413 & 671.81 & 1830.41 & 2039.03 & 53.71 & 10.23 & 1.37 & 0.04 \\
\cline { 2 - 9 }$y$ & 54251284 & 739.43 & 54251.28 & 57530.03 & 168.14 & 5.70 & 42.54 & 0.12 \\
\hline
\end{tabular}

Tabela 5. Análise de variância do consumo de energia durante o deploy de dependências

\begin{tabular}{lrrrrr} 
& Grau de Lib. & Soma Quad. & Quad. Médio & \multicolumn{1}{c}{ Estatística F } & \multicolumn{1}{c}{ Valor P } \\
\cline { 2 - 6 } Tamanho APK & 1 & $9.91 \times 10^{10}$ & $9.91 \times 10^{10}$ & $1.94 \times 10^{32}$ & $<2 \times 10^{-16}$ \\
\hline Tempo (s) & 1 & $1.73 \times 10^{10}$ & $1.73 \times 10^{10}$ & $3.38 \times 10^{31}$ & $<2 \times 10^{-16}$ \\
\hline Largura banda & 1 & $4.13 \times 10^{5}$ & $4.13 \times 10^{5}$ & $8.07 \times 10^{26}$ & $<2 \times 10^{-16}$ \\
\hline Resíduos & 112 & 0.00 & 0.00 & &
\end{tabular}

Um detalhe que chama a atenção é o fato da potência elétrica média demandada pelo Handset $\mathrm{B}$ durante o processo de deploy de dependência se manter próximo em 3 dos 4 casos analisados. Isso sugere que, a princípio, o consumo energético do dispositivo móvel durante o deploy de dependências depende do tempo decorrido no processo. A exceção está no caso em que o arquivo a ser transmitido pela rede é grande, e a velocidade de rede é alta. Isso indica que a transmissão continuada em alta velocidade aumenta o consumo médio de energia por parte do dispositivo móvel [Barbera et al. 2013].

\section{Considerações finais}

Este trabalho descreveu e discutiu dois experimentos que foram realizados com o framework CAOS D2D para avaliar os impactos do offloading de processamento no tempo de 
execução e consumo de energia de dispositivos móveis.

No que diz respeito ao tempo de execução de tarefas em offloading, os resultados apontam que existem casos em que o offloading representa uma opção muito vantajosa em termos de ganhos de tempo - com ganhos médios de até $78,1 \%$ com relação à execução local - mas também existem casos em que a execução local é a melhor opção. Essa variação depende da complexidade da tarefa a ser delegada e do tamanho de seus argumentos. Em alguns casos o offloading para o Handset A apresentava os melhores tempos de execução, enquanto em outros o offloading demonstrava tempos de execução menores se realizado para o TV Box.

Quanto ao aspecto da economia de energia proporcionada pelo offloading, os resultados apresentados aqui demonstram casos em que o offloading permitiu economia de até $88,5 \%$; mas também casos em que a execução local foi a melhor opção, devido ao consumo adicional de energia necessário para transferir o objeto que encapsula método e argumento para o dispositivo servidor. Alguns resultados dos experimentos demonstram um caso em que o offloading para um dispositivo representava aumento no tempo de execução, porém com melhoria de consumo de energia.

Através dos experimentos, foi possível perceber que o tempo decorrido na injeção de dependências é geralmente próximo do tempo ideal, e que o consumo energético do dispositivo móvel durante a injeção de dependências cresce em função do tempo empregado na transmissão, o que é consequência do tamanho do arquivo APK da aplicação e da velocidade da rede sem fio.

Uma limitação do CAOS D2D a ser trabalhada futuramente é a conectividade entre dispositivos, atualmente suportando apenas redes padrão 802.11. A introdução de padrões como Wi-Fi Direct ou Bluetooth pode aumentar as possibilidades de casos que se beneficiem do offloading, e novos experimentos dentro desses cenários podem ser realizados.

Um trabalho interessante a ser considerado é a avaliação de diferentes estratégias de injeção de dependência, como por exemplo, o uso de um repositório central de dependências, em vez da injeção direta a partir do dispositivo móvel cliente. Testes e características de ambas as abordagens podem ser analisados de forma a verificar se uma estratégia é melhor do que a outra; ou ainda, se uma abordagem híbrida é uma boa opção.

\section{Referências}

Abolfazli, S., Sanaei, Z., Sanaei, M. H., Shojafar, M., and Gani, A. (2016). Mobile cloud computing. Encyclopedia of Cloud Computing, page 29.

Artail, A., Frenn, K., Safa, H., and Artail, H. (2015). A framework of mobile cloudlet centers based on the use of mobile devices as cloudlets. In Advanced Information Networking and Applications (AINA), 2015 IEEE 29th International Conference on, pages 777-784. IEEE.

Barbera, M. V., Kosta, S., Mei, A., and Stefa, J. (2013). To offload or not to offload? the bandwidth and energy costs of mobile cloud computing. In INFOCOM, 2013 Proceedings IEEE, pages 1285-1293. IEEE. 
Chaffey, D. (2016). Mobile marketing statistics compilation. http://www.smartinsights.com/mobile-marketing/mobile-marketing-analytics/mobilemarketing-statistics/. Acessado em 09/10/2017.

Cuervo, E., Balasubramanian, A., Cho, D.-k., Wolman, A., Saroiu, S., Chandra, R., and Bahl, P. (2010). Maui: Making smartphones last longer with code offload. In MobiSys 2010, Proceedings ACM, pages 49-62, New York, NY, USA. ACM.

Dinh, H. T., Lee, C., Niyato, D., and Wang, P. (2013). A survey of mobile cloud computing: architecture, applications, and approaches. Wireless Communications and Mobile Computing, 13(18):1587-1611.

Fernando, N., Loke, S. W., and Rahayu, W. (2013). Mobile cloud computing. Future Gener. Comput. Syst., 29(1):84-106.

Ferrari, A., Giordano, S., and Puccinelli, D. (2016). Reducing your local footprint with anyrun computing. Computer Communications, 81:1-11.

Flores, H., Sharma, R., Ferreira, D., Kostakos, V., Manner, J., Tarkoma, S., Hui, P., and Li, Y. (2017). Social-aware hybrid mobile offloading. Pervasive and Mobile Computing, $36: 25-43$.

Ghasemi-Falavarjani, S., Nematbakhsh, M., and Ghahfarokhi, B. S. (2015). Contextaware multi-objective resource allocation in mobile cloud. Computers \& Electrical Engineering, 44:218-240.

Gomes, F. A., Rego, P. A., Rocha, L., de Souza, J. N., and Trinta, F. (2017). CAOS: A Context Acquisition and Offloading System. In 2017 IEEE 41st Annual Computer Software and Applications Conference (COMPSAC), pages 957-966. IEEE.

Gonçalves, G. D., Vieira, A. B., da Silva, A. P. C., and Almeida, J. M. (2016). Trabalho colaborativo em serviços de armazenamento na nuvem: Uma análise do dropbox. In XXXIV Simpósio Brasileiro de Redes de Computadores e Sistemas Distribuídos (SBRC).

Júnior, A. M. S., Sousa, M. L., Xavier, F. Z., Xavier, W. Z., Almeida, J. M., Ziviani, A., Rangel, F., Avila, C., and Marques-Neto, H. T. (2016). Caracterizacao do serviço de táxi a partir de corridas solicitadas por um aplicativo de smartphone. In XXXIV Simpósio Brasileiro de Redes de Computadores e Sistemas Distribuídos (SBRC).

Kolias, C., Ahlawat, S., Ashton, C., et al. (2013). Openflow-enabled mobile and wireless networks. White Paper.

Liao, L., Qiu, M., and Leung, V. C. (2015). Software defined mobile cloudlet. Mobile Networks and Applications, 20(3):337-347.

Santos, G., Trinta, F., Rego, P., and Gomes, F. (2017). CAOS D2D: Uma solução para offloading de métodos entre dispositivos móveis. In XVI WFA, pages 164-168.

Satyanarayanan, M. (1993). Mobile computing. Computer, 26(9):81-82.

Satyanarayanan, M., Bahl, P., Caceres, R., and Davies, N. (2009). The case for vm-based cloudlets in mobile computing. IEEE Pervasive Computing, 8(4):14-23.

Teo, C. L. V. (2012). Hyrax: Crowdsourcing Mobile Devices to Develop Proximity-Based Mobile Clouds. PhD thesis, Carnegie Mellon University, Pittsburgh. 